\title{
Key aspects of surface plasmon resonance spectroscopy for analytical chemistry applications
}

\begin{abstract}
The principles of the physical chemistry of surface plasmon resonance in metallic nanostructures have been briefly discussed. The application of this surface spectroscopy to the measurement of the concentration of chemical species is provided, after the exposure of the analytical chemistry detection strategies, the last one based on the consequences of the equation of the extinction spectrum derived from Gustav Mie's theory. Finally, the results of the detection of insecticides (dichlorvos and lindane) using nanoparticles of $\mathrm{Au}, \mathrm{Ag}$ and $\mathrm{Au}-\mathrm{Ag}$ alloy were reported as key examples to illustrate the advantages of synergistically combining the optical and surface properties of metals when forming alloys.
\end{abstract}

Keywords: plasmon, spectroscopy, nanoparticles, alloys, analytical chemistry

\author{
Volume 7 Issue 4 - 2018
}

\author{
Lorean Madriz, ${ }^{1,2}$ Ronald Vargas ${ }^{3}$ \\ 'Analysis and Catalysis, ${ }^{2}$ Electroanalysis, ${ }^{3}$ Electrochemistry \\ laboratories, Department of Chemistry, Simón Bolívar \\ University, Venezuela
}

\begin{abstract}
Correspondence: Lorean Madriz, Analysis and Catalysis and Electroanalysis laboratories, Simón Bolívar University, Caracas, Venezuela,Tel 5802 I2 906 3955, Email Imadriz@usb.ve
\end{abstract}

Received: February 26, 2018 | Published: July 26, 2018

\section{Introduction}

Metals have been of great interest in many areas as structural materials, but when they are converted into nanoparticles, they acquire different properties among which are their small size, large surface area and high adsorption capacity, ${ }^{1-3}$ which makes them promising for several analytical applications such as clinical, pharmaceutical, food and environmental. One of the most important aspects of metallic nanoparticles are their optical properties, which depend on their dimensions, shape, metal composition and surrounding environment. ${ }^{1-6}$ These properties are due to the principle of resonance of the surface plasmon (SPR) by which the electrons in the conduction layer of the material oscillate due to the frequency of electromagnetic radiation of the incident light. ${ }^{1-6}$ For this reason, in this mini review, the principles of surface plasmon resonance in metal nanostructures according to the Theory of Mie as well as the applications of this principle for the detection of analytes of interest will be considered, taking as an example the determination of dichlorvos and lindane by nanoparticles of $\mathrm{Au}, \mathrm{Ag}$ and their alloys in order to illustrate the synergistic effect of using the latter.

\section{Principles of surface plasmon resonance}

Surface plasmon resonance (SPR) is used to measure binding events between molecules ranging from ions to viruses. ${ }^{1}$ Current technology provides molecular binding with information on kinetics, affinity and very sensitive concentration measurements, with key applications in surface-enhance spectroscopy and sensing. ${ }^{4}$ The SPR is related to the physical process that involves electromagnetic waves beyond the diffraction limit and up to the nanoscale. In general, the SPR phenomenon involves the formation of a polariton, which is a coherent oscillation that arises from the interaction of electromagnetic waves with the collective oscillations of free electrons on the surface of a metal. ${ }^{2,4}$ The surface plasmons are mainly limited by the size and shape of the metal structure that supports the plasmon and by the environment that defines the metal-dielectric interphase: the refractive index and the nature and concentration of the chemical species. ${ }^{2}$

On the basis of linear optics, the dielectric function of a material reflects key information about the interaction between its electrons and light, and it should be noted that the ability of a metal nanoparticle to support a surface plasmon depends on its dielectric function, which is an optical function in a complex variable where both the real and the imaginary part correlate with the refractive index of the material and its light absorption coefficient, the latter varies with the wavelength of excitation of the light. In general, materials with negative real and small positive imaginary dielectric constant, can experiment the SPR phenomenon. ${ }^{4}$

Figure 1A illustrates the local surface plasmon resonance mode (LSPR), in which the electromagnetic wave induces a force on the electrons of the metal conduction band and promotes its oscillations, nanostructures with dimensions smaller than the wavelength of the light support LSPR phenomenon. Figure 1B shows the propagation surface plasmon resonance mode (PSPR), in this case the metal structures have at least one dimension that approaches the excitation wavelength, and then the plasmons propagate for distances of the order of $10-100 \mu \mathrm{m}$ along the metal-dielectric interphase. ${ }^{4}$ Silver, gold, lead, copper, tin, aluminum and alloys make from these metals support the plasmon resonance phenomenon from the UV-Visible regions to the near IR of the electromagnetic spectrum. ${ }^{3,5}$

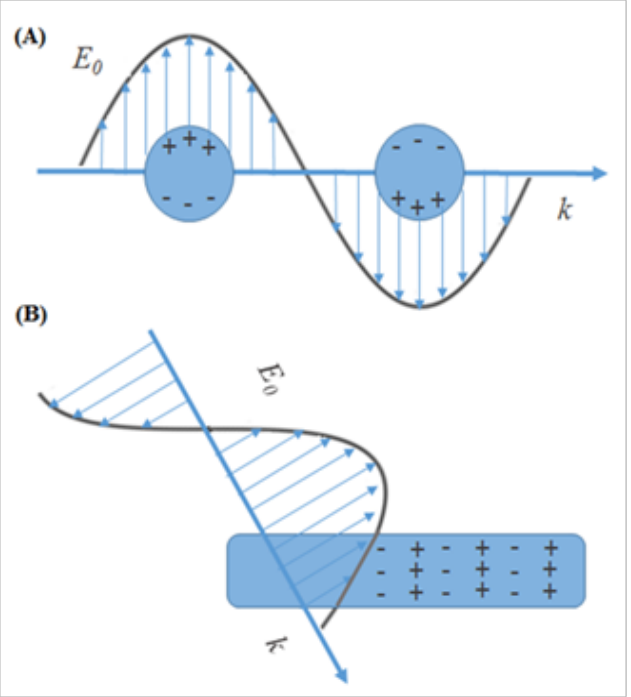

Figure I Scheme of light-matter interaction for the occurrence of plasmon resonance. (A) local surface plasmon resonance mode (LSPR) and (B) propagation surface plasmon resonance mode (PSPR). 


\section{Extinction spectrum and the analytical chemistry detection strategies}

Following the ideas of Gustav Mie for the resolution of Maxwell's electromagnetic equations applied to very small conductive spheres, illuminated with z-polarized light at a wavelength greater than the characteristic length of the particles, it is possible to find the extinction (absorption+scattering) spectrum $\left(E_{(\lambda)}\right)$ of the particles immersed in a dielectric, ${ }^{4,5}$ see equation (1). For a detailed mathematical treatment of the problem it is suggested to read the mandatory review of Willets and Van Duyne and take a special attention to their supplementary material. ${ }^{4}$

$$
E_{(\lambda)}=\frac{24 \pi N a^{3} \varepsilon_{\text {out }}^{3 / 2}}{\lambda \ln (10)}\left\{\frac{\varepsilon_{i(\lambda)}}{\left[\varepsilon_{r(\lambda)}+\chi \varepsilon_{\text {out }}\right]^{2}+\varepsilon_{i(\lambda)}{ }^{2}}\right\}
$$

where: $a$ is the characteristic length (size) of the metal particles, $\lambda$ is the wavelength of the light excitation, $N$ is the density particles number, $\varepsilon_{\text {out }}$ is the dielectric constant of the environment of the metal particles, $\varepsilon_{r(\lambda)}$ is the real part of the dielectric function of the metal, $\varepsilon_{\mathrm{i}(\lambda)}$ is the imaginary part of the dielectric function of the metal and $\chi$ is the aspect factor of the nanoparticles (for a sphere: $\chi=2$ ). ${ }^{4}$

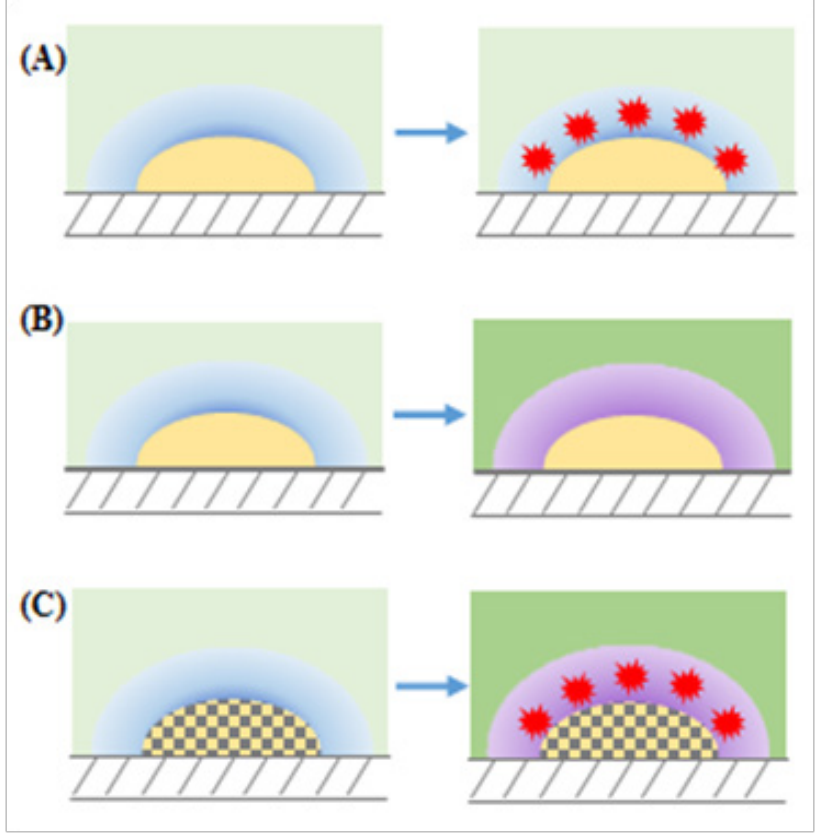

Figure 2 Analytical chemistry detection strategies: (A) molecular adsorption on metal particle surface, (B) refractive index change of the bulk solution by the increment of chemical species concentration and (C) synergic combination of optical and surface properties of metals when they form alloys: changes of the refractive index of the phases and molecular adsorption enhances.

For chemical analysis, a simple interpretation of equation 1 suggests that the spectrum of the system depends mainly on the refractive index of the medium, which is related to the concentration of the chemical species, as well as, the dielectric properties of the metal particle, which depends on their chemical nature, size and shape. $^{2-6}$

According to this theory the wavelength maximum in the SPR spectrum $\left(\lambda_{\max }\right)$ results sensitive to the dielectric constant of the phases, then changes in the local environment, as for example the adsorption of the chemical species on the metal surface, cause a shift in $\lambda_{\max }$ that results proportional to the change in refractive index induced by the adsorbate, and the last should be correlate with the concentration of the analyte. The change in the wavelength maximum $\left(\Delta \lambda_{\max }\right)$ results as follow:

$$
\Delta \lambda_{\max }=m \Delta n\left(1-e^{\frac{-2 d}{l_{d}}}\right)
$$

where: $m$ is the bulk refractive index response of the nanoparticle, $\Delta n$ is the change in refractive index induced by the adsorbate, $d$ is the effective adsorbate layer thickness, and $l_{\mathrm{d}}$ is the electromagnetic field decay length.

Figure 2 summarizes these effects and illustrates the commonly used analytical chemistry detection strategies based on SPR phenomenon. Figure 2A indicates the SPR change by molecular adsorption on metal particle surface, Figure 2B illustrates the plasmon relation with the refractive index change of the bulk solution by the increment of chemical species concentration and Figure 2C shows that the synergic combination of optical and surface properties of different metals when they form alloys, can be used to changes the refractive index of the phases and enhances the molecular adsorption.

\section{Chemical analysis application}

Noble metals have been commonly used for fabrication of nanostructures which have led to advances in several areas of science; moreover, these nanostructures have been employed in construction of sensors based on different principles in order to be used in specific applications. ${ }^{1}$ One of the principles is SPR, which was described previously and allow avail the optical properties of materials for detect molecular interactions near the nanoparticle surface through shifts in the spectral peak.

One way to increase the specificity of the sensors based on SPR, consist in modify it with biomolecules in order to detect interactions: biotin - streptavidin, antibody - antigen, nucleic acid hybridization, protein - carbohydrate, cytochrome - inhibitor, aptamer - protein, toxin - receptor, ${ }^{3,7-10}$ etc. Either sensors and biosensors, have applications in detection of drugs, anabolic steroid, opioids, stimulants and some peptide hormones in human organism, ${ }^{11,12}$ health, ${ }^{13-15}$ bacterias, ${ }^{16,17}$ viruses, ${ }^{18,19}$ allergens, ${ }^{20,21}$ pesticides, ${ }^{13,22,23}$ industrial safety, ${ }^{24,25}$ quality control, ${ }^{26-28}$ etc. The applications for sensing are very extends, please for more details see recent review of Boken et al. ${ }^{1}$ and the earlier review of Mayer and Hafner. ${ }^{3}$

As example of SPR detection, a particular case will be described, that is the use of screen printed graphite electrodes electrochemically modified with nanoparticles of $\mathrm{Au}, \mathrm{Ag}$ and $\mathrm{Au}$ - Ag alloy in determination of dichlorvos and lindane. The first is an organophosphorus insecticide used mainly in storage areas and granaries, its use has been generalized due to its relatively low persistence (months) in the environment compared to other compounds of similar application, however, they have very acute toxicity and exposure to high levels can affects the nervous system; ${ }^{29}$ and the last is an organochlorine insecticide used to protect crops from pests, it is even used in lotions and shampoos to treat lice and scabies, its use is restricted around the world, due to its neurotoxicity and because it is carcinogenic and mutagenic. ${ }^{30}$ The importance of 
this studying lies in that the insecticides have been used to control pests and weeds, and their residues affect environment and human being, moreover, the traditional methods of detection such as gas and/or liquid chromatography are expensive, consume very time and required specialized operator. ${ }^{31}$

Figure 3 shows the LSPR reflectance spectrum (A) and the wavelength maximum shift $v s$. concentration for dichlorvos measurements (B), both on $\mathrm{Au}, \mathrm{Ag}$ and $\mathrm{Au}-\mathrm{Ag}$ alloy nanoparticles electrocrystalized on screen printed graphite electrodes from DropSens $^{\circledR}$ (for synthesis details, please see the works of Liu et al. ${ }^{32}$ and Tao et $\mathrm{al}^{28}$ ). Instrumentation: for electrocrystallization a Bipotentiostat DropSens $\mu$ STAT200 and for spectrum measurements an Ocean Optics spectrometer s1024dw were used.

From Figure 3A, it can be highlighted the signals of surface plasmon of gold $(530 \mathrm{~nm})$, silver $(408 \mathrm{~nm})$ and the band of gold silver alloy $(488 \mathrm{~nm})$ between the other ones, which shown combined properties of both metals, the well-defined and intense plasmon of silver and the high surface properties of gold. ${ }^{3}$ It should be noted that, the SPR signal measurements are agree with the reported for

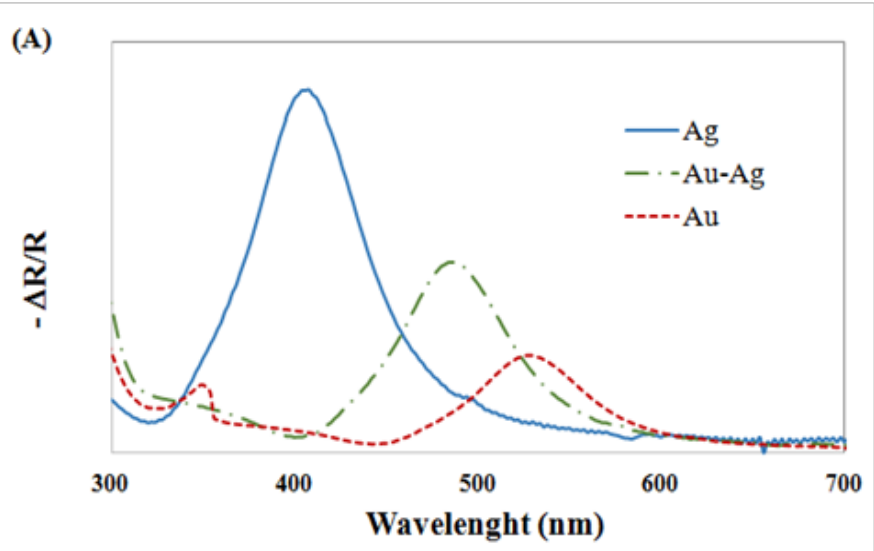

nanoparticles in the literature, ${ }^{9}$ and based on light scattering software from ScattPort, the corresponding sizes have been estimated: $41 \mathrm{~nm}$ for $\mathrm{Au}, 38 \mathrm{~nm}$ for $\mathrm{Ag}$ and $47 \mathrm{~nm}$ for $\mathrm{Au}-\mathrm{Ag}$ (1:1). These signals can be used for analytical detections.

Figure 3B shows the shift of the SPR wavelength maximum during the interaction of metal - particles with the insecticide. The detection based on SPR of dichlorvos yielded analytical responses with the following figures of merit: Limit of detection: $10 \mathrm{ppb}$, sensitivity: $2.0806 \mathrm{~nm} / \mathrm{ppm}$, linear range: $2-16 \mathrm{ppm}$ and standard deviation: 2 , these values indicate the efficiency of the principle described, because these are comparable to the reported literature using other sensing methodology and allow detect the insecticide into the interest range for environmental and health risks in municipal and industrial wastewater. ${ }^{33}$ On the same way, this strategy was also used for the detection of lindane, allowing its analytical validation through the following figures of merit: Limit of detection: $50 \mathrm{ppb}$, sensitivity: $0.7279 \mathrm{~nm} / \mathrm{ppm}$, linear range $0.01-1 \mathrm{ppm}$ and standard deviation: 3 . Finally, it should be noted that $\mathrm{Au}-\mathrm{Ag}$ alloy is reported as key example that illustrates the advantages of synergistically combining the optical and surface properties of metals when forming alloys.

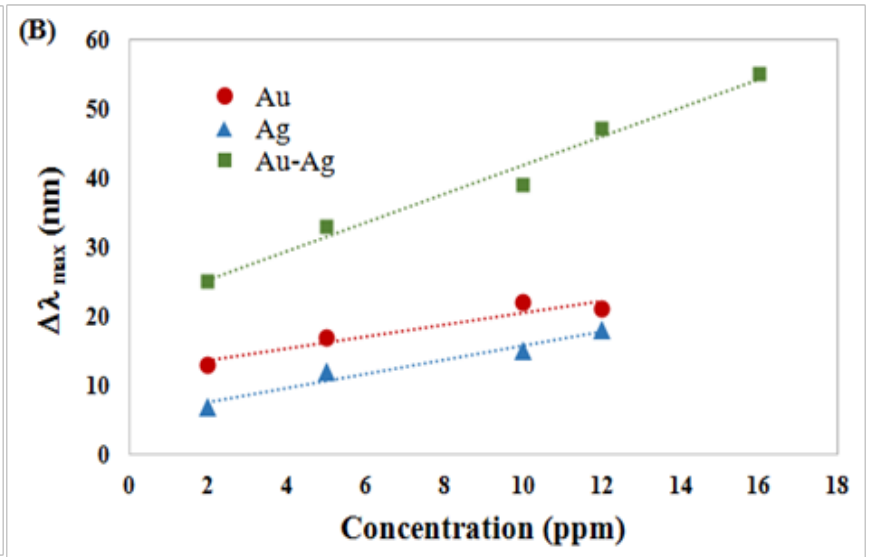

Figure 3 (A) LSPR reflectance spectrum and (B) Wavelength maximum shift vs. concentration for dichlorvos measurements, both on $\mathrm{Au}, \mathrm{Ag}$ and $\mathrm{Au}-\mathrm{Ag}$ alloy nanoparticles electrocrystallized on screen printed graphite electrodes (DropSens ${ }^{\circ} \mathrm{DRP}-\mathrm{CI} I 0$ )

\section{Conclusion}

A briefly revision about the principle of plasmonic resonance spectroscopy was discussed and the strategies to use sensors based on this effect were raised, the new sensing opportunities arise from this effect were commented. The synergic effect of optical and surface properties of alloy metals was exemplify using the detection of insecticides as dichlorvos and lindane on $\mathrm{Au}-\mathrm{Ag}$ alloy nanoparticles, which gave results into their range of interest for environmental and health risks in water solutions.

\section{Acknowledgements}

None.

\section{Conflict of interest}

The author declares that there is no conflict of interest.

\section{References}

1. Boken J, Khurana P, Thatai Sh, et al. Plasmonic nanoparticles and their analytical applications: A review. Applied Spectroscopy Reviews. 2017.
2. Rycenga M, Cobley CM, Zeng J, et al. Controlling the synthesis and assembly of silver nanostructures for plasmonic applications. Chem Rev. 2011;111(6):3669-3712.

3. Mayer KM, Hafner JH. Localized surface plasmon resonance sensors. Chem Rev. 2011;111(6):3828-3857.

4. Willets KA, Van Duyne RP. Localized surface plasmon resonance spectroscopy and sensing. Annu Rev Phys Chem. 2007;58:267-297.

5. Mulvaney P. Surface plasmon spectroscopy of nanosized metal particles. Langmuir. 1996;12:788-800.

6. Noguez C. Surface plasmons on metal nanoparticles: The influence of shape and physical environment. The Journal of Physical Chemistry C. 2007;111:3806-3381.

7. McPhillips J, Murphy A, Jonsson MP, et al. High-performance biosensing using arrays of plasmonic nanotubes. ACS Nano. 2010;4(4):2210-2216.

8. Dahlin AB, Chen S, Jonsson MP, et al. High-resolution microspectroscopy of plasmonic nanostructures for miniaturized biosensing. Analytical Chemistry. 2009;81(16):6572-6580.

9. Hiep HM, Yoshikawa H, Tamiya E. Interference localized surface plasmon resonance nanosensor tailored for the detection of specific biomolecular interactions. Analytical Chemistry. 2010;82(4):1221-1227. 
10. Guo LH, Chen GN, Kim DH. Three-dimensionally assembled gold nanostructures for plasmonic biosensors. Anal Chem. 2010;82(12):51475153.

11. Malekzad H, Zangabad PS, Mohammadi H, et al. Noble meta nanostructures in optical biosensors: basics, and their introduction to anti-doping detection. Trends in Analytical Chemistry. 2018;100:116135 .

12. Steinke N, Rio M, Wuchrera R, et al. Detection of diclofenac molecules by planar and nanostructured plasmonic sensor substrates. Sensors and Actuators B: Chemical. 2018;254:749-754.

13. Cho H, Baker BR, Wachsmann-Hogiu S, et al. Aptamer-based SERRS sensor for thrombin detection. Nano Lett. 2008;8(12):4386-4390.

14. Shankaran DR, Gobi KV, Miura N. Recent advancements in surface plasmon resonance immunosensors for detection of small molecules of biomedical, food and environmental interest. Sensor and Actuators B: Chemistry. 2007;121:158-177.

15. Thatai S, Khurana P, Prasad S, et al. Trace colorimetric detection of $\mathrm{Pb}^{2+}$ using plasmonic gold nanoparticles and silica-gold nanocomposites. Microchemical Journal. 2016;124:104-110.

16. Meeusen CA, Alocilja EC, Osburn WN. Detection of E. coli O157: H7 using a miniaturized surface plasmon resonance biosensor. Transactions of the American Society of Agricultural Engineers. 2005;48(6):24092416.

17. De Lorenzis E, Manera MG, Montagna G, et al. Erratum to 'SPR based immunosensor for detection of Legionella pneumophila in water samples. Optics Communications. 2013;294:420-426.

18. Zeng C, Huang X, Xu J, et al. Rapid and sensitive detection of maize chlorotic mottle virus using surface plasmon resonance-based biosensor. Analytical Biochemistry. 2013;440(1):18-22.

19. Lu X, Dong X, Zhang K, et al. A gold nanorods-based fluorescent biosensor for the detection of hepatitis B virus DNA based on fluorescence resonance energy transfer. Analyst. 2013;138(2):642-650.

20. Muller-Renaud S, Dupont D, Dulieu P. Quantification of $\beta$-casein in milk and cheese using an optical immunosensor. J Agric Food Chem. 2004;52(4):659-64.

21. Mohammed I, Mullett WM, Lai EPC, et al. Is biosensor a viable method for food allergen detect ion? Analytical Chimica Acta. 2001;444:97-102.
22. Qu F, Zhou X, Xu J, et al. Luminescence switching of CdTe quantum dots in presence of $\mathrm{p}$-sulfonatocalix[4]arene to detect pesticides in aqueous solution. Talanta. 2009;78:1359-1363.

23. Funari R, Della Ventura B, Schiavo L, et al. Detection of parathion pesticide by quartz crystal microbalance functionalized with UVactivated antibodies. Anal Chem. 2013;85(13):6392-7.

24. Chen KJ, Lu CJ. A vapor sensor array using multiple localized surface plasmon resonance bands in a single UV-vis spectrum. Talanta. 2010;81(4-5):1670-5.

25. Karakouz T, Vaskevich A, Rubinstein IJ. Polymer-coated gold island films as localized plasmon transducers for gas sensing. The Journal of Physical Chemistry B. 2008;112(46):14530-14538

26. Mack NH, Wackerly JW, Malyarchuk V, et al. Optical transduction of chemical forces. Nano Letters. 2007;7(3):733-737.

27. Jiang H, Markowski J, Sabarinathan J. Near-infrared optical response of thin film ph-sensitive hydrogel coated on a gold nanocrescent array. Opt Express. 2009; 17(24):21802-7.

28. Tao H, Lin Y, Yan J, et al. A plasmonic mercury sensor based on silver - gold alloy nanoparticles electrodeposited on indium tin oxide glass. Electrochemistry Communications. 2014;40:75-79.

29. Chen XM, Lin Z, Cai J, et al. Electrochemiluminiscence detection of dichlorvos pesticide luminol-CTAB medium. Talanta. 2008;76:10831087.

30. Anu Prathap M, Srivastava R. Electrochemical reduction of lindane $(\gamma-\mathrm{HCH})$ at $\mathrm{NiCo}_{2} \mathrm{O}_{4}$ modified electrode. Electrochimica Acta. 2013;108:145-152.

31. Zhang D, Liang $\mathrm{P}, \mathrm{Yu} \mathrm{Z}$, et al. The effect of solvent environment toward optimization of SERS sensors for pesticides detection from chemical enhancement aspects. Sensors and Actuators B: Chemical. 2018;256:721-728

32. Liu H, Favier F, $\mathrm{Ng} \mathrm{K}$, et al. Size-selective electrodeposition of meso-scale metal particles: A general method. Electrochimica Acta. 2001;47:671-677.

33. EPA. Method 622 The Determination of Organophosphorus Pesticides in Municipal and Industrial Wastewater. USA: Environmental Protection Agency; 1992. p. 1-21. 